\title{
Varones adolescentes en la Ciudad de Buenos Aires: barreras de género en la prevención y atención de la salud
}

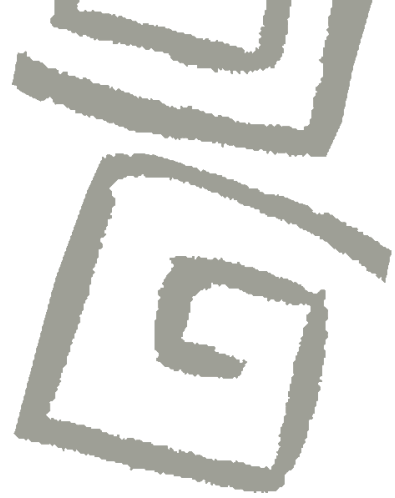

\author{
Adolescent males in the City of Buenos Aires: gender- \\ based barriers to health care and prevention
}

Débora Judith Tajer', Graciela Beatriz Reid², María Eugenia Cuadra ${ }^{3}$, Mónica Solís ${ }^{4}$, Juliana Fernández Romeral5 ${ }^{5}$, Lucía Daniela Saavedra ${ }^{6}$, María Laura Lavarello ${ }^{7}$, Rocío Paola Fabbio ${ }^{8}$

'Doctora en Psicología. Profesora Adjunta Regular, Facultad de Psicología, Universidad de Buenos Aires; Ciudad Autónoma de Buenos Aires, Argentina. $\bowtie$ (iD)

2Magíster en Psicoanálisis. Facultad de Psicología, Universidad de Buenos Aires; Ciudad Autónoma de Buenos Aires, Argentina. $\square$ (iD

${ }^{3}$ Magíster en Género, Sociedad y Políticas. Investigadora Facultad de Psicología, Universidad de Buenos Aires; Ciudad Autónoma de Buenos Aires, Argentina. $\square$ (iD)

${ }^{4}$ Licenciada en Psicología. Investigadora, Facultad de Psicología, Universidad de Buenos Aires; Ciudad Autónoma de Buenos Aires, Argentina. $\triangle$ (iD)

${ }^{5}$ Licenciada en Psicología. Investigadora, docente, Facultad de Psicología Universidad de Buenos Aires, Ciudad Autónoma de Buenos Aires, Argentina. $\square$ (iD)

${ }^{6}$ Licenciada en Psicología. Becaria, docente e investigadora, Facultad de Psicología, Universidad de Buenos Aires; Ciudad Autónoma de Buenos Aires, Argentina. $\triangle$ (iD)

${ }^{7}$ Licenciada en Psicología. Investigadora, Facultad de Psicología, Universidad de Buenos Aires; Ciudad Autónoma de Buenos Aires, Argentina. $\triangle$ (iD)

${ }^{8}$ Licenciada en Psicología. Investigadora, Facultad de Psicología, Universidad de Buenos Aires; Ciudad Autónoma de Buenos Aires, Argentina. $₫$ (iD)
RESUMEN La presente investigación surge a partir de identificar la dificultad para incluir las experiencias y necesidades de los varones adolescentes en el diseño de los modelos clínicos dirigidos a esa franja etaria y etapa vital. Por tal motivo, se decidió indagar la dimensión subjetiva de género en las prácticas que el sector salud dirige a la prevención y atención de la salud de los varones adolescentes, con el objetivo de visibilizar si estas se convierten en barreras para el avance en este campo. Para ello, se realizaron entrevistas semiestructuradas a 21 profesionales de distintas disciplinas integrantes de equipos de salud y a 14 varones adolescentes usuarios del sistema de salud de la Ciudad Autónoma de Buenos Aires; además se implementaron dispositivos grupales de indagación a adolescentes varones estudiantes de escuela secundaria, y se realizaron entrevistas en profundidad a informantes claves. Se ha identificado que las propias dinámicas institucionales no responden de modo adecuado a los riesgos que enfrentan los varones adolescentes -dentro de los cuales las construcciones hegemónicas de género cumplen un importante papel- ya que están ancladas en paradigmas biopolíticos de exclusión.

PALABRAS CLAVES Género y Salud; Masculinidad; Adolescente; Servicios de Salud del Adolescente; Argentina.

ABSTRACT This study is based on our previous work, in which we found evidence of the difficulties in including the experiences and needs of adolescent males in the design of clinical models aimed at that age group. We identified a need to explore the subjective dimension of gender in the practices of the healthcare sector directed at adolescent males, in order to determine if they constituted barriers to advancement in this field. Semistructured interviews were conducted with 21 professionals from different disciplines who made up healthcare teams, as well as 14 adolescent males using healthcare services in the City of Buenos Aires. Additionally, group techniques were employed with adolescent male secondary school students, and in-depth interviews were conducted with key informants. We were able to determine that institutional dynamics do not adequately respond to the risks faced by adolescent males - for whom hegemonic constructions of gender play an important role - given that they are based on exclusionary biopolitical paradigms.

KEY WORDS Gender and Health; Masculinity; Adolescents; Adolescent Health Services; Argentina. 


\section{INTRODUCCIÓN}

A partir de los resultados obtenidos en una investigación anterior titulada "Equidad de género en la calidad de atención en adolescencia" ${ }^{\prime(1,2)}$ se identificó la dificultad para incluir las experiencias y necesidades de los varones adolescentes en el diseño de los modelos clínicos dirigidos a esa franja etaria y etapa vital, en tanto la dimensión subjetiva de género no se encontró suficientemente visibilizada y transversalizada en las prácticas que el sector salud dirige a la prevención y atención de los varones adolescentes. En dicha investigación, se evidenciaron inequidades de género en la calidad de atención de la salud adolescente, por ejemplo, en el hecho de que las mujeres continúan siendo quienes más utilizan el sistema de salud y los equipos profesionales tienen poco impacto en el cambio de situación. Esto es solidario con los valores de género tradicionales que operan a nivel social y que el sector salud reproduce, en tanto se promueve la lógica del cuidado del cuerpo solo para las mujeres adolescentes e incluyen secundariamente a los varones ${ }^{(2)}$.

La pregunta que guía la investigación actual está centrada en la invisibilidad de componentes de la dimensión subjetiva de género en las prácticas en salud dirigidas a la prevención y atención de varones adolescentes. En este sentido, la relación entre los servicios de salud y los usuarios varones podría traducirse en distintas barreras de accesibilidad para estos últimos.

Los objetivos de la investigación están orientados, por una parte, a actualizar el estado del arte sobre la salud de los varones adolescentes desde una perspectiva de género, salud colectiva y subjetividad; $y$, por otra, a producir insumos para establecer acciones preventivas en este campo desde una perspectiva del derecho a la salud y género, con el fin de poder utilizarlos en estrategias de capacitación del personal de salud en este ámbito. De esta manera, se espera proporcionar conocimiento útil para la prevención y el desarrollo de intervenciones efectivas y pertinentes como también producir herramientas de transversalización de género en las políticas públicas en salud dirigidas a la prevención, promoción y atención de la salud de varones adolescentes, con el propósito de impactar en las etapas vitales siguientes.

De estos objetivos se desprenden distintas acciones u objetivos específicos, como identificar y analizar los imaginarios profesionales acerca de los varones adolescentes, sus necesidades y sus tipos de demandas en salud.

Las y los profesionales no suelen tener registro consciente cuando actúan desde sus imaginarios profesionales, entendiendo a estos como universos de sentidos o naturalizaciones que rigen los sistemas de creencias, certezas teóricas y/o técnicas que configuran los valores, sensibilidades, criterios éticos y estéticos, pautas conductuales y/o actitudinales de su hacer/pensar cotidiano del oficio en un determinado momento histórico-cultural Es relevante distinguir dichos imaginarios puesto que, en general, las y los profesionales creen que están operando solo desde sus conocimientos científicos. Es relevante también identificar y analizar si las prácticas de las y los profesionales incluyen la consideración de los riesgos específicos de los varones adolescentes -desde un abordaje integral que incorpore la perspectiva de género y subjetividad- en la identificación de los problemas epidemiológicos, los modos de consulta, los modelos de prevención y de atención.

A su vez, interesa identificar los imaginarios acerca de los riesgos específicos y las prácticas de los varones adolescentes en su vida cotidiana en la medida que tendrán impacto en su salud integral y en su interacción con el sistema de salud; como también, poder identificar y analizar desde la perspectiva de varones adolescentes usuarios y potenciales usuarios, la respuesta del sistema sanitario frente a sus problemas de salud.

A diferencia de anteriores publicaciones realizadas por el equipo(3), en este artículo se incluyen resultados preliminares obtenidos a partir del análisis de datos relevados por tres técnicas de indagación: entrevistas semiestructuradas, entrevistas en profundidad a informantes claves y dispositivos de indagación grupales. 
La investigación "Barreras de género en la prevención y atención de la salud de los varones adolescentes: significados y prácticas de usuarios y de profesionales de la salud" se enmarca en la programación UBACyT 17/19. Esta línea de investigación da continuidad a estudios anteriores, realizados por el mismo equipo, que indagaron la equidad de género en la calidad de atención en la niñez y la adolescencia.

\section{Estado actual de conocimiento sobre el campo de problemáticas estudiado}

Según refieren diversos autores pioneros en el campo de las masculinidades ${ }^{(4,5)}$, los varones, "supuestos sabidos" en la conformación del sujeto universal de la Modernidad, resultan aún desconocidos en sus prácticas y necesidades reales. Si bien desde hace varios años se aprecian avances en la producción científica sobre salud y masculinidad ${ }^{(6,7,8,9)}$, la comprensión de la problemática de la vulnerabilidad en varones y de los procesos de salud-enfermedad-cuidado, aún no incluye integralmente sus experiencias y sus dificultades específicas, más allá del impacto de la esfera del trabajo o, en el caso de adolescentes, la preparación para ingresar a esa esfera a futuro ${ }^{(9,10,11)}$.

Según De Keijzer(10), existe un modelo hegemónico de masculinidad que produce una subjetividad esencialmente dominante que se expresa en la discriminación y subordinación de las mujeres y otros varones que no se adaptan a dicho modelo. Esta forma hegemónica de socialización representa algunas claras ventajas para el varón, pero el paso del tiempo y su estereotipamiento implican riesgos en materia de salud en tres vectores principales: a) riesgo hacia mujeres, niños y niñas: el cual se expresa en la violencia de género, el abuso sexual infantil, el embarazo impuesto y la falta de participación masculina en la anticoncepción; b) riesgo hacia otros varones: a través de la legitimación de la burla, la presión y la violencia; y c) riesgo para sí mismo: en tanto la temeridad se presenta como prueba de la masculinidad, aumenta el riesgo de accidentes desde la infancia. De esta forma, ciertos comportamientos masculinos, considerados legítimos y hasta "esperados", los sitúan en situaciones de vulnerabilidad como accidentes, consumo problemático de alcohol y drogas ${ }^{(12)}$, violencia o ausencia de cuidado del propio cuerpo ${ }^{(13)}$. Los roles estereotipados de género los llevan a negar sus problemas de salud, no mostrar su vulnerabilidad $^{(14)}$ y les dificultan pedir ayuda e incorporar medidas de autocuidado ${ }^{(10,15)}$.

La adolescencia representa un período clave en el ciclo de vida, durante el cual las conductas de riesgo y de protección se inician y tendrán un impacto general en la salud, por su proyección hacia la vida adulta ${ }^{(16,17,18)}$. En términos epidemiológicos, estudios realizados en la región evidencian que los varones adolescentes tienen una mortalidad superior que las mujeres, se involucran menos en acciones de cuidado primario, tienen niveles más altos de necesidades de salud no cubiertas, presentan menos probabilidades de tener una fuente usual de acceso a la salud, menos probabilidades de haber visitado a una o un profesional de la salud en los últimos 12 meses y más probabilidades de haber tenido una consulta en urgencias en los últimos 12 meses $^{(19)}$. Desde la adolescencia, los varones consultan menos en el sistema de salud y las campañas orientadas a ellos muestran bajo impacto en la adopción de conductas preventivas y en la consulta precoz por problemáticas de salud de mayor incidencia en esta población ${ }^{(20)}$.

En el mismo sentido, la mortalidad por causas externas -homicidios, suicidios y accidentes- representa una problemática en salud de alta importancia epidemiológica a nivel mundial y nacional, constituyendo la primera causa de muerte para los varones adolescentes y jóvenes de entre 15 y 25 años, con una tasa de 5,6 varones por cada mujer ${ }^{(21,22)}$. El suicidio constituye la segunda causa de muerte en Argentina, en la población de 15 a 24 años, con una razón varón/mujer de 4 varones por cada mujer ${ }^{(23)}$. Estudios internacionales muestran que, en el caso de varones adolescentes gays, este riesgo aumenta, asociado al estigma, la discriminación, las distintas formas de violencia que padecen (verbal, 
física y sexual), la falta de apoyo social y las barreras de acceso y uso de los servicios de salud, entre otros fenómenos ${ }^{(24)}$. Esto configura el fenómeno denominado "sobre-mortalidad masculina temprana"(25), el cual determina en gran parte- la diferencia en la esperanza de vida al nacer entre varones y mujeres ${ }^{(26)}$. Por ello, se ha vuelto imperativo priorizar la prevención del suicidio en los programas mundiales de salud pública y de políticas públicas, como también concientizar acerca del suicidio en tanto cuestión de salud pública ${ }^{(17)}$.

Con relación al campo de la salud sexual y reproductiva, en la construcción y el sostenimiento de la identidad, el desempeño sexual tradicional es un factor crucial para los varones adolescentes y los procesos reproductivos no son tan relevantes como la sexualidad $^{(13,14)}$. Por lo general, establecen escaso contacto con los servicios de salud, utilizándolos solo en caso de extrema urgencia y nunca preventivamente ${ }^{(27)}$. Muchos varones recaban información sobre infecciones de transmisión sexual a través de redes sociales, familiares cercanos y, como última opción, acuden al sistema de salud ${ }^{(28)}$. Esto evidencia la importancia de ayudarlos a desarrollar una consciencia procreativa, para verse a sí mismos como seres sexuales capaces de crear vida humana y para proteger su propia salud y bienestar, la de sus parejas y potenciales hijos e hijas ${ }^{(29)}$. Asimismo, se torna fundamental diseñar e implementar planes de salud general y de salud sexual y reproductiva integrales y especializados para esta población ${ }^{(30,31)}$.

La adolescencia también constituye un período de exploración y autodescubrimiento, en la que se desarrolla consciencia y entendimiento con respecto a la identidad de género y la orientación sexual. En la actualidad, categorías identitarias fijas o estables como "hetero" "gay" o "lesbiana" no resultan significativas para las y los adolescentes, quienes se definen en "fluidez" y prefieren no

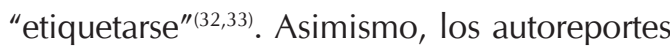
de identificación no se condicen de manera lineal con las prácticas y/o fantasías ${ }^{(34,35)}$. Pese a ello, actualmente, en el contexto nacional, se evidencia una tendencia a realizar diagnósticos cada vez más precoces de "disforia de género", especialmente en la adolescencia. Esta teoría no ha sido probada científicamente, sus bases ideológicas han sido impugnadas y criticadas por la población trans, puesto que no se encasillan en identificaciones fijas, sino que entienden su identidad como exploración y tránsito entre ambos géneros y otros alternativos, elementos que deben ser considerados por las y los profesionales de la salud para una adecuada atención de esta población ${ }^{(36,37)}$.

Se ha identificado que los costos físicos y emocionales de los ordenamientos de género dominantes no resultan evidentes para los adolescentes varones, y son pocos los espacios y las oportunidades que ellos tienen para expresar sus dudas y frustraciones o denunciar situaciones de violencia física y simbólica a las que se ven sometidos y, por lo general, no perciben cómo las representaciones de género afectan otras dimensiones de sus vidas ${ }^{(13)}$. Junto a lo anterior, en los servicios de salud se identifican diversos obstáculos como la falta de preparación de las y los profesionales de la salud para atender a los usuarios varones y la escasez y ausencia de modelos, programas y materiales específicos dirigidos, desde una perspectiva de género, hacia el cuidado y la prevención de la salud en varones adolescentes ${ }^{(3,13,14,38)}$.

Existe consenso internacional en afirmar la necesidad de incorporar la perspectiva de género, a lo largo del ciclo vital, en la promoción y la atención de la salud ${ }^{(39,40)}$, siendo la adolescencia un período fundamental. Incorporar una perspectiva de género implica considerar dos elementos centrales: a) especificidad de género: mirar las necesidades específicas que los jóvenes poseen en términos de salud y desarrollo por cuenta de su proceso de socialización, lo que significa, por ejemplo, involucrarlos en discusiones sobre el uso de drogas o comportamientos de riesgo, ayudarlos a procesar críticamente por qué se sienten presionados a comportarse de distintas formas, entre otras actividades; y b) equidad de género: involucrar a los varones en la discusión y reflexión sobre la jerarquía de género con el objetivo de llevarlos a asumir su parte de responsabilidad en el cuidado 
de las hijas y los hijos, en las cuestiones de la salud reproductiva y en las tareas domésticas ${ }^{(13)}$. En atención a ello, se vuelve necesario incorporar los aportes del campo de la subjetividad ${ }^{(41)}$, en sus dimensiones de género $^{(9,42)}$ y sumar como herramienta conceptual la noción de modos de subjetivación de género, aportada desde la articulación entre los estudios de género y el psicoanálisis ${ }^{(9,43)}$. Dicha noción es central para visualizar cómo la conformación temprana de valores, exigencias, legitimidades, modalidades de despliegue pulsional, estándares de autoestima diferenciales para varones y para mujeres ligados a los modos diferenciales por género en la construcción del psiquismo ${ }^{(44)}$, tienen efectos diferenciales en los procesos de salud-enfermedad-cuidado de quienes pertenecen a cada uno de los colectivos genéricos ${ }^{(45)}$.

Las políticas públicas de promoción de la salud pierden impacto en la población de adolescentes varones al no considerar las modalidades propias en que la masculinidad hegemónica, como ideal, conduce a los varones a participar de los riesgos en los cuales se ven involucrados ${ }^{(9)}$. La posibilidad de que los adolescentes abandonen un comportamiento riesgoso está relacionada estrechamente con la posibilidad de que se le ofrezcan alternativas de satisfacción valiosas para ellos ${ }^{(16)}$. Uno de los desafíos actuales es la transferencia de los resultados de estos estudios a los sistemas de salud para su incorporación en los modelos de prevención y atención de los varones adolescentes, desafío que esta investigación intenta abordar. Esto permitirá mejorar las políticas preventivas en el corto y en el mediano plazo, así como lograr mayor eficiencia en el largo plazo, dado que, entre otras cuestiones, prevenir es menos costoso en términos económicos que curar ${ }^{(1)}$.

\section{Hipótesis de la investigación}

Como se anticipó en la introducción, la pregunta que guía la investigación está centrada en la invisibilidad de componentes de la dimensión subjetiva de género en las prácticas en salud dirigidas a la prevención y atención de varones adolescentes. En este sentido, la relación entre los servicios de salud y los usuarios varones podría traducirse en distintas barreras de accesibilidad para estos últimos, por lo que se parte de los siguientes supuestos que se desprenden de las producciones e investigaciones realizadas por este equipo y la revisión bibliográfica recabada:

- Podrían existir sesgos de género en la identificación de los modos diferenciales de los varones adolescentes de enfermar, de consultar y de ser atendidos, que operarían como barreras en la prevención y atención de esta población específica.

- La ausencia de perspectiva de género en los modelos de atención y abordaje de la salud en población masculina adolescente podría invisibilizar la complejidad de sus necesidades bio-psico-sociales específicas y diferenciales.

- Los sesgos de género estarían presentes en los modos de comunicar las medidas preventivas, lo cual limitaría la eficacia de la prevención de los riesgos específicos en varones adolescentes.

- Podría existir una falta de identificación y de promoción de la detección precoz de "la masculinidad como factor de riesgo", que se iría consolidando a partir de la etapa adolescente y expondría a los varones a riesgos y vulnerabilidades específicas ligadas a la masculinidad hegemónica en etapas vitales posteriores.

\section{METODOLOGÍA}

\section{Enfoque de la investigación y diseño metodológico}

La revisión de la bibliografía especializada muestra una falta de innovación metodológica en términos de los diseños de investigación y un énfasis en estudios de carácter epidemiológico ${ }^{(24)}$, por lo que existen escasas investigaciones cualitativas y participativas con el propósito de aumentar la comprensión de los fenómenos problematizados con los adolescentes. Además, la mayoría de los 
estudios realizados con población adolescente presentan un fuerte sesgo adultocéntrico, en el que más bien se habla "sobre" ellos con diferentes adultos y no "con ellos de ellos"(46,47). Justamente, uno de los antecedentes relevados en el Proyecto UBACyT 2013/16 "Equidad de género en la calidad de atención en adolescencia" muestra la falta de implicación y la posición pasiva de los varones adolescentes respecto del cuidado de su propia salud y la de otras y otros. En razón de lo mencionado, se justifica la realización de un estudio de carácter cualitativo ${ }^{(48)}$ y participativo ${ }^{(49)}$ que fomente una posición de sujetos con capacidad de agencia, para contribuir a la construcción de políticas públicas y programas que reconozcan a las y los adolescentes y jóvenes en términos de ciudadanía y con capacidad para posicionarse como actores estratégicos ${ }^{(16,39,40)}$.

La presente investigación utiliza un tipo de diseño exploratorio y comprensivo, para contribuir a ampliar un campo de estudio poco explorado, así como abierto, flexible y emergente. De tal forma, se adapta a medida que se genere nuevo conocimiento sobre el fenómeno estudiado. La muestra es de carácter intencional y no probabilística.

\section{Participantes y técnicas de indagación}

El trabajo de campo abarca, en esta etapa, un total de 21 entrevistas a profesionales de distintas disciplinas y 14 entrevistas a varones adolescentes usuarios de un servicio de adolescencia de un hospital público, un centro de salud y acción comunitaria y un hospital de comunidad de la Ciudad Autónoma de Buenos Aires. Asimismo, se han llevado a cabo dos dispositivos grupales de indagación a adolescentes varones estudiantes de una escuela secundaria pública.

Las y los participantes de la investigación que configuran las unidades de análisis son profesionales de la salud, varones usuarios del sistema de salud de 15 a 19 años y alumnos varones de escuela secundaria de 15 a 19 años. La selección de este grupo etario responde a la hipótesis respecto de las escasas consultas de varones adolescentes en el sistema de salud, y la necesidad de ampliar la información con la que cuentan los varones adolescentes de la población general, sus imaginarios sociales acerca de su propia salud y la respuesta del sistema de salud.

El trabajo de campo se viene llevando a cabo en la Ciudad de Buenos Aires, desde el año 2018, en los servicios de adolescencia de cinco efectores de salud: dos hospitales públicos, dos centros de salud y acción comunitaria -correspondientes al primer nivel de atención- y un hospital de comunidad. Asimismo, se desarrollaron dispositivos grupales de indagación en una escuela secundaria pública y próximamente se iniciará el proceso de entrevistas en una escuela privada.

La recolección de datos se realizó a través de entrevistas semiestructuradas a profesionales de equipos de salud en adolescencia y varones usuarios del sistema de salud; dispositivos grupales de indagación a adolescentes varones estudiantes de escuela secundaria; y entrevistas en profundidad a informantes claves.

En las entrevistas a profesionales de equipos de salud en adolescencia se buscó que identificaran puntos críticos en los mensajes preventivos y de promoción de la salud, así como aspectos de los dispositivos de atención de salud adolescente que se constituyeran en barreras de accesibilidad material y simbólica al sistema de salud de los varones adolescentes. Las personas entrevistadas, consideradas informantes claves, fueron seleccionadas intencionalmente con el criterio de que sean referentes en los ejes de indagación en esta investigación, sea por su ámbito de especialidad, de acción o de responsabilidad. Cabe aclarar que los ejes que aborda la presente investigación son: 1) Epidemiología diferencial; 2) Consumo problemático; 3) Violencia; 4) Nutrición; 5) Salud sexual y reproductiva; y 6) Identidad de género.

A partir de estas entrevistas se buscó conocer los avances y desafíos actuales en el abordaje preventivo y de tratamiento de varones adolescentes desde una perspectiva de estudios de las masculinidades y género; la situación local en el enfoque preventivo y de tratamiento de esta población, y la valoración 
de la incorporación de la perspectiva de género; y la dimensión de la subjetividad en la prevención y atención de la salud de los varones adolescentes.

Las entrevistas semiestructuradas individuales realizadas a los usuarios varones adolescentes tuvieron como objetivo principal identificar los motivos de consulta y cuál era la frecuencia con la que los jóvenes asistían al sistema de salud. Se exploró su valoración respecto de los modos de atención de las y los profesionales que habían consultado y si recibieron orientación respecto a cómo mantenerse saludables. Se indagaron imaginarios acerca de los riesgos a los que estaban expuestos con relación a su salud, si se consideraban a sí mismos como susceptibles de padecer algún problema de salud o accidente asociado a alguna actividad recreativa, y el grado de conocimiento sobre mensajes de prevención en torno a los problemas de salud.

Además, a partir de su participación se buscó conocer sus percepciones e imaginarios sobre barreras materiales y/o simbólicas en el acceso a la atención frente a los problemas de salud; y obtener datos sociodemográficos e información relativa a su inscripción educativa.

En el diseño metodológico se incluyó el uso de dispositivos de indagación grupal a estudiantes secundarios varones -pensados como usuarios o potenciales usuarios del sistema de salud- como un modo de sortear las dificultades de encontrarlos en los servicios. Se buscó relevar cuándo concurrían, cuáles eran los motivos de consulta y la frecuencia en que asistían al sistema de salud, como así también qué tiempos consideraban razonables para realizar una consulta. También se indagó acerca de la respuesta que recibían de parte de las y los profesionales cuando realizaron la consulta, la percepción de los riesgos a los que estaban expuestos con relación a su salud, si se consideraban a sí mismos como susceptibles de padecer algún problema de salud o accidente con relación a alguna actividad recreativa, deportiva u otras. Se intentó indagar, además, los imaginarios sobre sexualidad, salud sexual, procreación, consumos problemáticos; las acciones en relación con el conocimiento de sus propios problemas de salud; qué percepción tenían de los mensajes preventivos dirigidos a los varones de su edad, sobre la relación entre formas de vida o conductas y sus riesgos en salud, brindados por las y los profesionales, los medios de comunicación y organismos gubernamentales; y qué percepción poseían sobre barreras materiales y/o simbólicas en el acceso a la atención de su salud. En este artículo se presentarán los resultados preliminares de las entrevistas individuales y de los dispositivos grupales en instituciones educativas.

\section{Consideraciones éticas del estudio}

Para resguardar los aspectos éticos de la investigación, con cada una de las personas entrevistadas, se implementó un consentimiento informado que contempló las recomendaciones del protocolo de Helsinki en las investigaciones en salud humana. Asimismo, cabe señalar que la presente investigación cuenta con el aval ético de la Universidad de Buenos Aires desde el momento en que se aprobó el proyecto.

Teniendo en cuenta que en la investigación participan adolescentes, la ética de la investigación es clave desde el diseño hasta la ejecución total del proyecto ${ }^{(50)}$. En tanto se trata de una investigación inclusiva y participativa, implica también la protección de los derechos de las y los adolescentes durante su realización ${ }^{(46,51)}$. En razón de ello, el diseño de las herramientas de indagación se realizó a partir de identificar y solucionar aspectos y dilemas éticos implicados, como el equilibrio de la diferencia de poder entre el equipo de investigadoras e investigadores y los adolescentes participantes. Se incluyó el uso de consentimientos informados para adolescentes y para adultos a cargo, según corresponde desde el paradigma de autonomía progresiva. Estos se adaptaron a las características específicas de los participantes, considerando y respetando su eventual negativa a participar o a continuar la investigación. En todo momento se tomaron acciones orientadas a resguardar la voluntariedad, confidencialidad y anonimato. 
Mediante acuerdos con los servicios de salud y con el uso del consentimiento informado se logró el acceso a entrevistar usuarios varones adolescentes, a quienes se brindó información específica sobre el estudio y se solicitó su participación. Al realizar la entrevista en la sala de espera del servicio de adolescencia correspondiente, se tomaron las consideraciones pertinentes a fin de lograr privacidad; se acordó interrumpir la entrevista al ser llamado a la consulta y, si estaba dentro de las posibilidades y/o intereses del entrevistado, se retomaba luego de la consulta.

En los dispositivos de indagación grupal en la escuela pública, mediante el uso de consentimientos informados diseñados a tal fin, se convino el encuadre de trabajo con autoridades, docentes y estudiantes varones, de modo de garantizar los mismos principios.

\section{Actividades de transferencia de conocimientos}

Como se mencionó, uno de los objetivos de esta investigación se vincula a la transferencia de resultados a los servicios de salud. A tales fines, se planean realizar talleres con las y los profesionales de los servicios y centros de salud que hayan participado de este proyecto. Asimismo, parte de los insumos de esta investigación y un estudio anterior de este equipo (Proyecto UBACyT 13/16 "Equidad de Género en la Calidad de Atención en Adolescencia"), se están utilizando para la realización de diversas actividades en escuelas medias y preuniversitarias de la Ciudad Autónoma de Buenos Aires. En estas actividades se abordan problemáticas que emergen en el ámbito educativo: situaciones de acoso y conductas sexuales abusivas de los adolescentes hacia sus compañeras y los "escraches" que las jóvenes realizan a fin de visibilizar dichas situaciones ${ }^{(52)}$. Asimismo, se ha participado en espacios de intercambio profesional en un centro de salud mental y en el ámbito académico, donde la dimensión subjetiva de género está siendo visibilizada e interrogada en el abordaje de problemáticas en la adolescencia.
Para finalizar, las actividades en el espacio de docencia que sostiene este equipo de investigación se ven continuamente retroalimentadas con los aportes del trabajo de campo, por lo que se promueve la actualización de las y los estudiantes de psicología que cursan la materia "Introducción a los Estudios de Género" en la Facultad de Psicología de la Universidad de Buenos Aires, en temas vinculados a salud, salud mental, género y subjetividad.

\section{RESULTADOS PRELIMINARES}

A continuación, se desarrollan los primeros resultados de los ejes: epidemiología diferencial, consumo problemático, salud sexual y reproductiva, y nutrición, que fueron obtenidos a partir de las entrevistas realizadas a las y los profesionales de la salud. También se analizan los primeros datos obtenidos en las entrevistas individuales con usuarios del sistema de salud y en los dispositivos de indagación grupal con varones que asisten a una escuela pública de la Ciudad Autónoma de Buenos Aires.

\section{Los varones adolescentes y su presencia/ausencia en las consultas de salud}

Las y los profesionales que participaron de las entrevistas identifican mayoritariamente que las consultas de los adolescentes varones en los servicios de salud son muy poco frecuentes, refieren que solo consultan por el "apto médico", certificado de salud solicitado por las instituciones educativas y deportivas. Algunas y algunos profesionales aprovechan esta demanda como "oportunidad ganada" para llevar adelante un examen de salud que los jóvenes no demandarían espontáneamente. Los condicionantes sociales del "ser varón" y el rol de las mujeres en los cuidados de la salud (alta presencia en las consultas de madres y parejas mujeres como aquellas que los llevan o acompañan) son dos respuestas que los 
profesionales brindaron con mayor frecuencia. Uno de ellos indica que:

...el condicionante social de que los varones son más fuertes, que necesitan menos controles, que no es de machito ir al médico: va al médico el que tiene problemas o es débil, eso también es un condicionante muy fuerte, cada vez más fuerte. (Pediatra, hospital de comunidad)

De la información preliminar de las entrevistas a los adolescentes se desprende la poca presencia de varones que consultan, cuestión que se tradujo en algunas dificultades y obstáculos encontrados para el abordaje del trabajo de campo. Por ello, se decidió ir a buscarlos en el ámbito educativo donde, debido a la alta tasa de escolaridad de la Ciudad Autónoma de Buenos Aires, se les pudo consultar por sus experiencias en el ámbito de la salud.

$\mathrm{Al}$ entrevistar a los usuarios sobre las consultas que realizan responden, de forma mayoritaria y en consonancia con lo expresado por los profesionales, que llegan al sistema de salud por el "apto físico". Sin embargo, se destaca que una minoría de los usuarios refieren consultar por chequeos generales o problemáticas específicas:

...consulto en el sentido de cómo estoy, por si tengo algo, por si me llega a pasar algo, por eso vengo. Los tratamientos que hago, de vez en cuando, son los chequeos en la sangre y esas cosas. (17 años, usuario de hospital público)

...hago consultas por el tema de la columna, la piel, a veces también veo a un dermatólogo, también hago consultas por el tema del oído. (16 años, usuario de hospital de comunidad)

En la escuela, los varones refieren que son sus madres quienes insisten en que realicen consultas médicas y se registra la mayor realización de consultas de control en quienes cuentan con obra social (prestación de atención médica para trabajadores y trabajadoras en relación de dependencia).
Las respuestas de los usuarios y de las y los profesionales orientan a pensar que las consultas de los varones aparecen mediadas por mujeres. Cuando dicha mediación no se produce, las consultas disminuyen, y las que aparecen son en su mayoría por la solicitud de "apto físico", y en menor medida por cuestiones físicas como consecuencia de accidentes, por un lado, y cuestiones tanto estéticas como deportivas, por el otro. Es decir, podría pensarse que, mayoritariamente, la construcción de la demanda al servicio de salud se ubica de forma externa: los varones van a consultas llevados o acompañados por mujeres, por pedido de una institución o por una dolencia o accidente físico de gran magnitud. Esto evidenciaría la falta de implicación y la posición pasiva de los varones adolescentes respecto del cuidado de su propia salud.

El otro modo de presencia masculina adolescente en el sistema de salud se produce en el servicio de guardia debido a traumatismos y urgencias varias, relacionadas con la exposición a situaciones riesgosas. Las y los profesionales identifican los accidentes, los consumos problemáticos de alcohol y drogas, la violencia y la ausencia de cuidado del propio cuerpo como comportamientos predominantemente masculinos con graves consecuencias para la salud. Estas acciones y conductas legitimadas como parte de la masculinidad social, los sitúan en situaciones de vulnerabilidad específica ${ }^{(53)}$.

En este sentido, al consultar a los adolescentes usuarios sobre la exposición a accidentes y situaciones de pelea, la mayoría refiere que se debe a una cuestión inherente al "ser varón", ligado a mandatos sociales:

Sí, he escuchado muchas veces, a mí mismo me han dicho que si alguien me pega yo se la tengo que devolver [...] porque si no te dicen que sos un buchón, un cagón. (16 años, usuario de hospital de comunidad)

Los hombres tienden más a ser más agresivos y entonces solucionan las cosas de una forma agresiva. Es por eso... Igual las mujeres también. (16 años, usuario de centro de salud y acción comunitaria) 
Los usuarios mayoritariamente reconocen estar expuestos y exponerse a situaciones de violencia, tanto ellos como sus amigos o conocidos, pero identifican que las mujeres tampoco están exentas. Con base en lo relevado resulta interesante retomar estas respuestas en particular, en las que los usuarios parecen poner en discusión el estereotipo de femineidad tradicional ${ }^{(9)}$.

Por otro lado, en la escuela los varones refirieron que la exposición a estas situaciones "depende de la persona". Se hace foco en la individualidad y no reconocen la violencia como conducta asociada al mandato de la masculinidad hegemónica. Otros adolescentes dieron cuenta de la exposición a peleas, por ejemplo, en el deporte: "peleo en el club". De esta manera, aparece el rol del deporte en el devenir de la masculinidad ${ }^{(54)}$ en tanto organizador de la identidad depositado en el resguardo del "honor a la camiseta", la pertenencia a un grupo/equipo y la rivalidad con aquellos que no pertenecen a este.

En las respuestas de los usuarios se destaca el componente de la fuerza física y de "poner el cuerpo" al servicio de la pelea como un modo de accionar para legitimar y/o resguardar la masculinidad hegemónica. En este sentido, De Keijzer ${ }^{(10)}$ sostiene que la masculinidad hegemónica implica tres vectores de riesgo. El riesgo hacia otros varones y el riesgo hacia sí mismos son los dos que se pondrán de relieve. El primero es el riesgo hacia los pares mediante la legitimación de acciones violentas, haciendo de estas una condición necesaria, aunque no suficiente, del estereotipo masculino. Y el segundo denota la contracara del primero: en tanto la masculinidad hegemónica implica un riesgo hacia otros varones, también implica un riesgo hacia sí mismos. Es decir, "poner el cuerpo" al servicio de acciones violentas también implicaría "ofrecer el cuerpo" para dar testimonio de su masculinidad, por ende, oscilar entre ser el riesgo y ponerse en riesgo, donde el cuidado del propio cuerpo pasa a un segundo plano.

En esta línea, al consultarles a las y los profesionales si las prácticas incluyen la consideración de los riesgos específicos de los varones adolescentes en los modelos de atención y de prevención, identificaron dificultades para hacer efectivos los mensajes preventivos y de promoción de la salud. Otro obstáculo señalado es la dificultad de generar dispositivos necesarios para el acceso y la atención de los varones adolescentes, lo cual se ve reflejado en la escasez de varones que consultan. Estas dificultades podrían estar operando como barreras materiales y simbólicas de accesibilidad al sistema de salud de los varones adolescentes. En este sentido, identifican el valor de la propuesta de disponer de consejerías y talleres en sala de espera, con el objeto de utilizarlo como dispositivos para concientizar a los varones y hacerlos participar activamente del cuidado de su salud, como modo de ampliar la accesibilidad. Asimismo, consideran que la existencia de mayor cantidad de médicos varones que se dediquen a la atención facilitaría que los adolescentes realicen consultas. $\mathrm{Al}$ respecto, uno de los profesionales entrevistados señaló: "Yo creo que tiene que estar disponible. Pero bueno, los sistemas de salud imponen situaciones. $Y$ en la atención primaria el perfil es netamente femenino" (Odontólogo, centro de salud y acción comunitaria). Esta afirmación evidencia ciertas condiciones estructurales que podrían operar como barreras a la accesibilidad de los varones adolescentes al sistema de salud: la feminización de la salud pública y, especialmente, de la atención primaria, la cual se asociaría tradicionalmente al rol de cuidado atribuido a las mujeres ${ }^{(55)}$.

Por lo anteriormente señalado, las y los profesionales identifican que existe una brecha entre lo que el sistema de salud valora que debiera ser un motivo de consulta de los varones adolescentes -ligado a los indicadores epidemiológicos de género y generación- y las razones efectivas por las cuales estos últimos acuden al sistema de salud. Por tal motivo, señalan la importancia de incluir la voz de los varones adolescentes al momento del diseño y definición de las políticas de atención que el sistema de salud fija para ellos. Esto implicaría, incluir información acerca de cómo quieren ser atendidos y cuáles identifican que son sus necesidades, para incorporarlas en 
las acciones de promoción, prevención, atención y cuidados. Al mismo tiempo, señalan la necesidad de que los adolescentes conozcan sus riesgos específicos en salud, de manera comprensible y que dicha información adquiera un sentido para ellos, teniendo en consideración que, en esta etapa del ciclo vital, las conductas de riesgo y protección que se constituyan tendrán un impacto general en la salud, por su proyección hacia la vida adulta( $^{(16,18,39)}$.

Con respecto al trato que reciben por parte de las y los profesionales, los adolescentes en general tienen buenas experiencias. En la escuela, refirieron que tener un familiar con trabajo registrado resulta un beneficio por el acceso a la obra social. En un taller, un adolescente expresó: "Ir al hospital, te la regalo", lo cual refiere no solo al acceso a la salud en términos de clase, sino que también evidencia la actual situación de precarización laboral de las y los trabajadores y la significación del hospital como lugar donde solo asisten las clases menos favorecidas. Los estudiantes hicieron referencia a cuestiones de índole económica que obstaculizan el acceso al sistema de salud. Aquí se mencionaron cuestiones desde el costo del pasaje del transporte público hasta las medidas de fuerza que se producen en un contexto de reducción del presupuesto para la salud pública ("están de paro"). Otro refirió: "hacer un control privado es muy caro para una boludez", lo cual lleva a inferir que, por el costo económico que implica, no realizan consultas si la problemática no tiene gravedad para ellos.

Asimismo, señalaron otras barreras: la espera de turnos en el sistema público y el tener que levantarse temprano para la obtención de un turno y/o ser atendidos: "tenes que ir a la madrugada a sacar turno", "para hacer un análisis te dan para un mes... y ya te morís [...] en algún momento te vas a morir". Es decir, esto implica no solo que en esta etapa la idea de muerte resulta lejana y no hay representación del riesgo o del cuidado de la salud en el presente, sino que los jóvenes identifican barreras concretas y estructurales del sistema de salud que obstaculizan su acceso.

\section{Las problemáticas con mayor prevalencia en la salud de los varones adolescentes}

Las y los profesionales que participaron de las entrevistas identifican que los problemas que representan mayor impacto en la salud de los varones adolescentes son: el consumo problemático de sustancias, las conductas de riesgo, la obesidad y la salud sexual y reproductiva.

\section{Aspectos vinculados al consumo problemático de sustancias y prácticas de riesgo}

Respecto del consumo problemático, en uno de los servicios donde se llevó adelante el trabajo de campo, consideran el alcohol como una "batalla" perdida por la salud pública y ganada por el mercado. Explican que este consumo está instalado masivamente en los adolescentes como una propuesta de época, mediante la cual se obtiene placer y es aprovechado para vencer la timidez, poder relacionarse e identificarse con un grupo de pertenencia y animarse a seducir. Esto también aparece en el discurso de un adolescente que, haciendo referencia a las campañas de prevención, señala:
...Es importante que haya mensajes así, que debería haber más, se fomenta mucho en Argentina las cervezas, las cervece- rías, se consume mucho en Argentina, y puede ocasionar muchos problemas el día de mañana y aunque hoy no nos demos cuenta puede ser grave. (18 años, usuario de hospital de comunidad)

Las y los profesionales consideran como problemático la naturalización por parte de las personas adultas, responsables de dicho consumo, frente al avance del mercado de bebidas alcohólicas y hayan asumido -a modo de estrategia de reducción de daños espontánea- que "la previa" -reunión de pares antes de una salida nocturna en la que suele consumirse bebidas alcohólicas- es mejor que la hagan en una casa con bebidas alcohólicas de mayor calidad que la que podrían Ilegar 
a consumir en otros lugares. Lo señalado no colabora en dirección a una propuesta de conductas saludables para las y los adolescentes, lo cual que se expresa con alto impacto en los indicadores de morbimortalidad de género y generación.

Uno de los indicadores del consumo problemático que consideran las y los profesionales es la función que cumple la sustancia para cada sujeto, lo cual determina el límite entre si se trata de un consumo problemático o recreativo/social. Aclaran que trabajan con estrategias de reducción de riesgos y daños, articulando con los servicios de salud mental y con centros especializados en la temática. En consonancia con lo expresado por los equipos de salud, los usuarios en su mayoría entienden que el consumo se vuelve problemático "cuando se vuelve costumbre" (17 años, usuario de hospital público), "cuando se consume constantemente" (15 años, usuario de hospital de comunidad), "cuando lo haces siempre" (15 años, usuario de hospital público) o "cuando se vuelve una necesidad" (16 años, usuario de hospital de comunidad).

Resulta interesante señalar que las preguntas referidas al consumo problemático de sustancias fueron mayormente respondidas por los adolescentes como problemas que tienen otros, en una asociación directa de lo problemático del consumo a situaciones de adicción. Ante las preguntas que buscaban relevar lo ocurrido en el marco de salidas o las referidas a situaciones personales o de personas cercanas, aparecieron relatos y asociaciones con situaciones de riesgo al conducir o de violencia.

Las y los profesionales también hacen referencia a lo que denominan consumos de élite o, en palabras de una profesional:

Son chicos que el alcohol como depresor les da sueño, les termina dando sueño, se sienten muy nauseosos, no quieren tomar o vomitan, pero el energizante los despabila, los despierta, les da cafeína, los vigoriza entonces siguen tomando. Son los "borrachos despiertos". (Médica especializada en adolescencia, hospital de comunidad)
Cabe señalar que, en este caso, predomina la combinación de psicofármacos, alcohol y drogas de diseño. Esta forma de consumo genera riesgos altísimos cuando los adolescentes llegan a la guardia ya que no se sabe cuál es la combinación de sustancias ingeridas y sus efectos pueden ser letales. Por otro lado, estos "policonsumos" conllevan a sostener prácticas de riesgo, entre las que se pueden destacar: abusos, sexo sin protección, acoso y violencias asociadas al modelo de masculinidad hegemónica.

En los adolescentes de mayor vulnerabilidad social se identifica una fuerte presencia de consumo de pasta base (paco) que es de alta toxicidad. $\mathrm{Y}$ aun cuando desde el sector salud se intenta que no tengan barreras de acceso al centro de salud, refieren poco impacto, dado que es muy baja la posibilidad de reducir el riesgo en estos adolescentes. En su mayoría, los usuarios refieren no conocer lugares de atención para los consumos problemáticos, pero sí conocen compañeros de escuela o personas cercanas que tuvieron o tienen problemas de consumo. Asimismo, les parece relevante recibir información sobre estos temas: uno de ellos refiere sobre los mensajes de prevención:

\footnotetext{
...me parece bien, lo podés encontrar en todos lados. Sobre todo, para gente que le da vergüenza hablar de esas cosas, es una buena opción. Por lo menos sabes dónde podés acudir. (16 años, usuario de hospital público)
}

\section{Aspectos vinculados a la salud sexual y reproductiva}

La mayoría de las y los profesionales de los servicios coinciden en que, estadísticamente, los varones adolescentes no consultan espontáneamente por estos temas. Las pocas consultas que se realizan aparecen cuando estos temas son introducidos por el personal de salud. De existir un motivo de consulta, está relacionado con un síntoma en los genitales, asociado generalmente con infecciones de transmisión sexual, lo cual se vería reflejado 
en la presencia de varones adolescentes en las consultas de los centros de prevención asesoramiento y diagnóstico del $\mathrm{VIH}$-sida, que funcionan tanto en hospitales públicos, como en centros de salud y organizaciones comunitarias, según refirió uno de los profesionales entrevistados.

Asimismo, se registran consultas cuando presentan dificultades para el desempeño sexual ligado a la penetración. Los procesos reproductivos -incluyendo su responsabilidad-son considerados como irrelevantes en comparación con el desempeño y rendimiento sexual $^{(13,14)}$. Esto evidenciaría la construcción de una sexualidad masculina en torno a valores tradicionales, asociada a prácticas en las cuales lo relevante sería la excitabilidad del varón en tanto emblema de la masculinidad hegemónica. De esta manera, el imperativo hacia la actividad conduce a una desestima del propio deseo, así como del deseo de quien sea partenaire. Ambos deseos caen bajo la presión por el logro ${ }^{(9,42)}$.

Las y los profesionales señalan que el hecho de que las campañas de salud sexual y reproductiva estén mayoritariamente dirigidas a las mujeres no colabora a incluir a los varones, quienes espontáneamente no se sienten convocados. Para superar estas dificultades y barreras de accesibilidad, una de las estrategias que utilizan es aprovechar cualquier consulta que realicen para abordar este tema. Al mismo tiempo que identifican que el escaso tiempo del cual dispone cada profesional con un paciente para abordar la diversidad de temas que incluye la historia clínica integral, resulta más escaso aún para motivar e informar a quienes se presentan más reticentes, evidenciando de qué manera la lógica mercantil estructura la atención en salud al privilegiar la "producción" en términos de cantidad de consultas atendidas. Es decir, se privilegia la eficiencia por sobre la eficacia. Otro de los profesionales refiere que:

Hay algo que a mí me preocupa que no se trabaja en salud sexual y reproductiva: que no se promueve la vasectomía. Siempre la mujer poniendo el cuerpo [...] los patrones médicos son machistas [...] Es un tema para trabajar con adolescentes en su futuro, que existe esa opción. No se trabaja la vasectomía e incluso existe fantasías de que te cortan los testículos. (Odontólogo, centro de salud y acción comunitaria)

De esta manera, el entrevistado hace referencia a los "invisibles" de la salud sexual y reproductiva abordada desde un enfoque tradicional que sería reproducido por la política pública en salud.

El centro de salud y acción comunitaria cuenta con una Red Matricial de Salud Sexual y Reproductiva que es interdisciplinaria. Dicho equipo armó un dispositivo de recepción espontánea de todas las consultas sobre el tema que funciona mañana y tarde. El personal de administración fue el primero en ser entrenado, ya que se lo concibe como "la puerta de entrada" al sistema de salud. Este equipo de salud sexual y reproductiva trabaja todos los días y las consultas son recibidas como "urgencias" del primer nivel de atención. Cuentan con una consejería en la que brindan información sobre el test de embarazo y métodos anticonceptivos o la continuidad si lo tuviera. Aún con este dispositivo inclusivo refieren que los varones solo representan el $2 \%$ de las consultas y el $98 \%$ son mujeres.

Al consultar a los usuarios si conocen las opciones anticonceptivas que ofrece el sistema de salud, mayoritariamente refieren conocer las pastillas anticonceptivas y los preservativos. Uno de ellos señala: "regalan anticonceptivos" (17 años, usuario de hospital de comunidad), lo cual resulta una respuesta minoritaria pero interesante para pensar no solo qué métodos ofrecen los servicios de salud, sino cómo los ofrecen, de qué manera los varones adolescentes reciben esa información y si comprenden que se trata de su derecho.

Los jóvenes refieren que el uso del preservativo es una decisión individual ligada al cuidado de su salud, pero al consultarles si los cuidados o los métodos son los mismos si la pareja sexual es ocasional o no, los varones adolescentes heterosexuales entrevistados piensan, dudan o afirman que la decisión 
puede ser compartida y aparece la opción de relegar el cuidado al uso de pastillas anticonceptivas por parte de su pareja.

Asimismo, resulta pertinente destacar que, aunque minoritariamente, varios adolescentes responden que no conocen cuáles son las opciones anticonceptivas que ofrece el sistema de salud. Estos resultados preliminares refuerzan que la anticoncepción y el cuidado es algo que sucede en el cuerpo de las mujeres, del cual los varones aparecen desligados, aunque desde los servicios de salud se ofrecen talleres que aprovechan la oportunidad de consulta durante el embarazo de las parejas o cuando las acompañan con su hijo y/o hija. Un profesional de salud mental (licenciado en Psicología, hospital público) refiere, como estrategia para incluir a los adolescentes en estas consultas, la realización de talleres para que los varones que van a ser padres puedan participar. Les entregan allí el texto de la Ley de Parto Humanizado a modo de involucrarlos. Las y los profesionales identifican dos barreras en la participación: por un lado, la falta de información y, por otro, la salida al mundo laboral que les impide acompañar a sus parejas.

Las y los profesionales participantes valoran la "escucha abierta", la entienden como una vía para que los usuarios tengan la confidencialidad asegurada, a la vez que les permite transmitir información y no "emitir órdenes" porque evalúan que atenta contra la adherencia. Intentan de este modo que los adolescentes participen activamente con aquello que les pasa y por lo que consultan.

Las personas entrevistadas señalan que es importante que en un servicio de adolescencia no haya solo ginecología, dado que esa presencia -cuando es la única especialidad médica presente- produce que los varones digan: "para qué voy a ir yo, si soy varón" (Médica especializada en pediatría y adolescencia, hospital público). En este sentido, nuevamente resaltan la importancia de la oferta de urólogos, hebiatras y que esté contemplada la presencia de médicos varones para aquellos que puedan sentir pudor de ser revisados por mujeres.

La mayoría de las y los profesionales refieren que, en la consulta, preguntan si el adolescente tuvo relaciones sexuales, a qué edad, cuántas parejas tuvo y si usa o no usa algún método anticonceptivo. Con los varones, se indaga si se encuentra en una relación sexo-afectiva y algunas características puntuales de esta ya que hay casos en los cuales pueden ser ellos los vulnerados. Esto puede suceder al iniciar relaciones bajo coerción, aunque es más común en el caso de las mujeres. En tanto que los varones usuarios consultados responden que, mayoritariamente, las y los profesionales les preguntan si iniciaron sus actividades sexuales, pero no les preguntan el género de la otra persona, dando por sentado que se trata de una mujer.

Uno de los profesionales entrevistados apuntó a la diferenciación entre orientación sexual y prácticas:

Hay ciertas preguntas que a veces resultan molestas ¿con quién tenes relaciones sexuales? [...] Muchas veces la gente responde con una mentira porque está pensando en el juicio de valor del profesional. La heteronorma tiene que estar en el papel [...] Pero indagar sobre el tipo de relaciones sexuales... es a las prácticas adonde hay que apuntar. (Odontólogo, centro de salud y acción comunitaria)

De esta manera, la pregnancia de la cis-heteronormatividad en las consultas sobre salud sexual y reproductiva -señalada por el profesional y los adolescentes consultadospodría funcionar como barrera de acceso, en tanto puede llevar a fallas en la atención médica generando errores en el diagnóstico de algún problema de salud, brindando información incorrecta sobre medidas de prevención y/o sosteniendo acciones de invisibilización, maltrato u otras formas de discriminación ${ }^{(56)}$.

$\mathrm{Al}$ consultarles a dónde o a quiénes recurren si tienen alguna inquietud sobre la sexualidad, la mayoría de los usuarios entrevistados busca alguien o algo que les inspire confianza, entre los que se mencionan: amigos, hermanos, padre, Internet, profesores o a su pareja. Estas respuestas refuerzan la hipótesis de que ciertas condiciones estructurales de las instituciones del sistema de salud podrían operar 
como barrera para su acceso en dos sentidos complementarios: por un lado, el sistema de salud se encuentra con dificultades para generar el acceso y la atención a los varones y, por otro lado, los varones suelen no considerar el sistema de salud como un espacio al cual recurrir ante dudas sobre su sexualidad o por prevención y atención. Al respecto, algunas y algunos profesionales refirieron que la escuela debe ser la gran protagonista en este tema a través de la implementación de la Ley de Educación Sexual Integral (ESI). En esta misma línea, en la escuela, los adolescentes señalaron que los talleres que recibieron sobre este tema se centraron en el embarazo y el uso del preservativo. Uno de ellos comentó: "no me gusta que hablen siempre de embarazos". Resulta relevante señalar que las y los estudiantes valoran la existencia de la ESI aunque son críticos de su implementación, en tanto resulta reducida a una serie de talleres o clases anuales.

\section{Salud alimentaria y problemáticas específicas}

Respecto de la obesidad como problemática de alto impacto en la salud de los adolescentes varones, las y los profesionales señalan que es un emergente de las condiciones de vida, determinada por las condiciones materiales de existencia.

En la mayoría de los varones con sobrepeso, observan que es mínimo el porcentaje que tiene compulsión por la comida o ansiedad por comer. Identifican que la mayoría vive en contextos familiares donde la mayor parte de sus integrantes son obesos u obesas y ello no se significa como un problema. Esto sucede con más frecuencia en familias en situación de vulnerabilidad social, en las cuales la alimentación se basa, principalmente, en el consumo de hidratos de carbono (fundamentalmente harinas).

Otro factor que favorece el desarrollo de esta problemática es el miedo o la inseguridad, que ha hecho que las familias valoren el barrio de residencia como inseguro y se "encierren". Al respecto, una profesional señala:
Desde la niñez están guardados por el miedo. Eso ya genera un sobrepeso que lo arrastran desde la niñez y lo recibimos en la adolescencia [...] están con el telefonito o la tablet que reciben en la escuela, comiendo y sentados. (Médica pediatra, hospital público)

Entonces, esta población está conformada por jóvenes sedentarios, conectados a dispositivos electrónicos, que realizan poca actividad física, limitados por el temor de las personas adultas a la inseguridad, sumado a la falta de recursos para ir a clubes, que se consideran lugares seguros.

En cuanto a la vigorexia es más común en los adolescentes de clase media y es una tendencia que va creciendo. La preocupación está centrada en el consumo de anabólicos en etapas donde los varones aún no han alcanzado el desarrollo total. Según las y los profesionales, los síntomas que presentan están relacionados con la elevación de enzimas producto del agotamiento muscular por el exceso de actividad física para aumentar la masa muscular o por la ingesta de anabólicos. Este interés podría ser puesto en relación con la construcción de corporalidades que denoten fortaleza y asociado a patrones estéticos masculinos estereotipados. Desde la perspectiva de la subjetividad, conlleva a la construcción del cuerpo en términos mecanicistas, es decir, como algo a ser entrenado, pero no como constitutivo de su subjetividad, con el cual los varones pueden entablar otro tipo de conexión ${ }^{(9,42)}$. De esta manera, el cuerpo solo merecería atención si "falla" de alguna manera. Esto explicaría, en parte, la escasa problematización de los adolescentes de la vigorexia -lo cual las personas entrevistadas refirieron como motivo de preocupación- y su alta presencia en las salas de urgencia, tal como fue expuesto anteriormente.

Los varones adolescentes entrevistados refieren que las y los profesionales abordan la salud alimentaria vinculada a alguna cuestión específica de salud o a las actividades deportivas: "yo hacía rugby, entonces me decían cómo alimentarme bien y esas cosas. Más que nada por cuestiones de deporte, 
hacer una rutina para que me mantenga bien" (16 años, usuario de hospital público). Otro refiere a su período de recuperación física "[me orientaron] a comer fruta. Más que nada por la rodilla, por los huesos... banana. $Y$ yendo a un gimnasio y haciendo 'gambas', bicicleta, más que nada bicicleta por la pierna" (17 años, usuario de hospital público). Sin embargo, la salud alimentaria no aparece como una cuestión por la que consulten específicamente, vinculada a la prevención y promoción, sino que aparece en relación a cuestiones deportivas o estéticas. Son las y los profesionales quienes introducen esta temática en las consultas.

\section{CONCLUSIONES}

A partir de la información relevada hasta el momento, se observa que las y los profesionales relacionan la presencia masculina adolescente en el sistema de salud con instancias riesgosas y que sitúan a esta población en situaciones de vulnerabilidad específica. Lo relevado coincide con lo manifestado por los jóvenes, quienes señalan varias cuestiones que se convertirían en barreras de acceso al sistema de salud: identifican una barrera de acceso administrativa en cuanto a la burocracia institucional en el acceso a los servicios, no se sienten convocados por temas de salud sexual y reproductiva debido a su abordaje tradicional (centrado en la prevención de embarazos), no consideran relevante realizar consultas de prevención, sino de atención ante una dolencia o enfermedad considerable, y reconocen que a veces llegan a los servicios de salud por su madre o su pareja.

A su vez, desestiman la importancia de los controles y acciones preventivas y cuando llegan a los servicios de salud lo hacen por cuestiones vinculadas a las actividades deportivas, su interés en componer alguna herida o mejorar su rendimiento, por cuestiones estéticas o cuando presentan una dolencia significativa que les impide desarrollarse en su cotidianeidad. En cuanto a la salud sexual y reproductiva, en general conocen dos métodos de cuidado: preservativo y pastillas. Destacan que se cuidan para prevenir embarazos o enfermedades, aunque no se sienten convocados directamente por la temática. Es relevante destacar aquí que no se mencionan prácticas que incluyan las diversidades sexuales y sus diferentes métodos de cuidado. Tampoco referencian a las instituciones de salud como un lugar al cual ir a consultar acerca de la sexualidad, sino que se apoyan en los padres, profesores o pares, con quienes "tienen confianza".

Sobre las situaciones de violencia, las reconocen, pero en su mayoría señalan que las mujeres también llevan a cabo acciones violentas. Esta información relevada indicaría cierta naturalización de la violencia, en tanto también se la adjudican a las mujeres sin dar cuenta de una reflexión sobre la relación que puede haber entre la violencia y el género masculino. Asimismo, y en tensión con lo antes mencionado, resulta interesante la observación que realizan, ya que permite pensar en las discusiones que aparecen entre ellos sobre los estereotipos de género femeninos tradicionales. En este contexto, se considera de vital importancia que las y los profesionales de la salud transversalicen un análisis de género -incorporando los factores subjetivos de dicha dimensión ${ }^{(9,41,42)}$ en las consultaspuesto que permitiría comprender con un mayor grado de integralidad las causas específicas de las problemáticas abordadas, como así también sus manifestaciones. Se visibiliza así de qué manera la conformación temprana de valores, exigencias, estándares de autoestima diferenciales por género poseen efectos específicos en los procesos de salud-enfermedad-cuidado de los adolescentes. En esta línea, considerar los requerimientos diferenciales por género en las campañas de prevención de las enfermedades no transmisibles contribuiría a reducir la morbilidad y mortalidad prematura en la población estudiada.

Las y los profesionales, que participaron de las entrevistas, ubicaron la importancia de incluir la voz de las masculinidades adolescentes al momento del diseño y definición de las políticas de atención del ámbito sanitario, ya que identifican la brecha existente entre lo que el sistema de salud valora que debiera ser un 
motivo de consulta de dicha población -ligado a los indicadores epidemiológicos de género y generación- y las razones efectivas por las cuales los jóvenes acuden al sistema de salud. Es decir, existe la inquietud por atender a los adolescentes pero, mayoritariamente, lo que el sistema de salud ofrece son dispositivos tradicionales de prevención y atención que hacen que los varones no accedan a estos y cuando sí lo hacen, no se les ofrece lo que requieren. La invisibilidad de los componentes subjetivos de género operaría estructurando barreras para el acceso en dos sentidos complementarios: por un lado, el sistema de salud se encuentra con dificultades para generar el acceso y la atención a los varones y, por el otro, los varones suelen no considerar al sistema de salud como un espacio al cual recurrir ante inquietudes sobre, por ejemplo, su sexualidad. Estas dificultades podrían estar operando como barreras de accesibilidad materiales y simbólicas al sistema de salud de los varones adolescentes, promoviendo inequidades.

Se han identificado esfuerzos por conmover dichas lógicas, a través de los talleres en sala de espera o los dispositivos territoriales que salen al encuentro de los varones en los espacios por los que circulan, por ejemplo, la escuela. Queda abierta la inquietud respecto a los espacios que pueden ser llevados adelante para adolescentes que no se encuentran escolarizados.

Asimismo, las y los profesionales identificaron que la escasa presencia de profesionales varones en los servicios de adolescencia y la sola existencia de ginecología como única especialidad ofrecida podrían funcionar como barrera a la accesibilidad de los jóvenes a la atención, evidenciando cómo la segregación por género en la estructuración del sistema de salud -en sus diferentes niveles- tendría efectos en los procesos de salud-enfermedad-atención.

Se ha identificado que las propias dinámicas institucionales no responden de modo adecuado a los riesgos que enfrentan los varones adolescentes -dentro de los cuales las construcciones hegemónicas de género cumplen un importante papel- ya que están ancladas en paradigmas biopolíticos de exclusión. A modo de ejemplo, surge de las entrevistas realizadas, el hecho de que las campañas de salud sexual y reproductiva están mayoritariamente dirigidas a mujeres, y no convoca a los adolescentes a adoptar prácticas de cuidado.

\section{AGRADECIMIENTOS}

La investigación en la que se basa este artículo, titulada "Barreras de género en la prevención y atención de la salud de los varones adolescentes: significados y prácticas de usuarios y de profesionales de la salud" y dirigida por la doctora Débora Tajer, ha sido evaluada, aprobada y financiada por la Universidad de Buenos Aires, como proyecto de investigación UBACyT 2017-2019, categoría grupo consolidado, consignada bajo el código 20020160100137BA. Se agradece al equipo de investigación que está conformado por Alejandra Lo Russo, Mariana Gaba, Victor Javier Forni, Elizabeth Ceneri, Federico Cappadoro, Germán Alvarenga, Jessica Gutman, Natalia Arlandi, Florencia Borello Taiana y Lucero Garber.

\section{REFERENCIAS BIBLIOGRÁFICAS}

1. Tajer D, Reid G, Ceneri E, Solís M. Equidad de género en la calidad de atención en adolescencia.
En: XXI Anuario de Investigaciones. T. 1. Buenos Aires: Facultad de Psicología, UBA; 2014. p. 233-240.

2. Tajer D, Reid G, Lo Russo A, Salvo Agoglia I. Equidad de género en la calidad de atención en adolescencia, Parte 2. En: XXII Anuario de Investigaciones. T. 1. Buenos Aires: Facultad de Psicología, UBA; 2015. p 301-308.

3. Tajer D, Reid G, Fernández Romeral J, Saavedra L. Barreras de género en la prevención y atención de la salud de los varones adolescentes. En: XXIV Anuario de Investigaciones. T. 1. Buenos Aires: Facultad de Psicología, UBA; 2018. p. 221-226.

4. Gilmore D. Hacerse hombre: Concepciones culturales de la masculinidad. Buenos Aires: Paidós; 1994.

5. Connel RW. La organización social de la masculinidad. En: Valdés T, Olavarría J, (eds.). Masculinidad/es: poder y crisis. Santiago de Chile: Ediciones de las Mujeres; 1997. p. 31-48. 
6. Giffin K. Esfera de reprodução em uma visão masculina: considerações sobre a articulação da produção e da reprodução, de classe e de gênero. Physis Revista de Saúde Coletiva. 1994;4(1):23-40. Disponible en: 10.1590/S0103-73311994000100002.

7. De Keijzer B. Hasta donde el cuerpo aguante: género, cuerpo y salud masculina. La Manzana, Revista Internacional de Estudios sobre Masculinidades. 2006;1(1).

8. Tajer D. Varones y enfermedad cardiovascular: Género y subjetividad en la construcción del riesgo en enfermedades cardiovasculares en varones jóvenes. I Coloquio Internacional de Estudios sobre Varones y Masculinidades y I Congreso Nacional de la Academia Mexicana de Estudios del Género de los Hombres: Violencia: ¿el juego del hombre? México; 2006.

9. Tajer D. Heridos corazones: Vulnerabilidad coronaria en varones y mujeres. Buenos Aires: Paidós; 2009.

10. De Keijzer B. El varón como factor de riesgo: Masculinidad, salud mental y salud reproductiva. En: Tuñón E, (coord.). Género y salud en el sureste de México. Villa Hermosa: ECOSUR-UJAD; 1997.

11. Bernales M. Hombres y conductas de riesgo en salud: hacia una comprensión de la complejidad sociocultural de la salud masculina. IV Coloquio Internacional de Estudios sobre varones y masculinidades: Patriarcado en el siglo XXI: Cambios y Resistencias. Santiago de Chile; 2015.

12. Jeifetz $V$, Tajer D. Equidad de género en la adherencia al tratamiento de adicciones: Representaciones y prácticas de profesionales y pacientes en un servicio de internación de un hospital público. En: XVIII Anuario de Investigaciones. T. 2. Buenos Aires: Facultad de Psicología, UBA; 2010.

13. PROMUNDO-ECOS. Sexualidad y salud reproductiva [Internet]. San Pablo: Promundo; 2015 [citado 29 mar 2019]. Disponible en: https://tinyurl. $\mathrm{com} / \mathrm{rkz} 3 \mathrm{~d} 7 \mathrm{u}$.

14. Garita Arce C. La construcción de las masculinidades: Un reto para la salud de los adolescentes [Internet]. San José de Costa Rica: Caja Costarricense de Seguro Social, PAIA, OPS, OMS, UFPA, Agencia Sueca de Desarrollo Internacional; 2001 [citado 29 mar 2019]. Disponible en: https:// tinyurl.com/w6wwxpn.

15. Aparicio Aviña G, Ibarra Casals D, Rodríguez F. Beneficios de abandonar los privilegios masculinos del sexismo. IV Coloquio Internacional de Estudios sobre varones y masculinidades: Pa- triarcado en el siglo XXI: Cambios y Resistencias. Santiago de Chile; 2015.

16. Pasqualini D, Llorens A, (comp.). Salud y bienestar de adolescentes y jóvenes: una mirada integral. Buenos Aires: Organización Panamericana de la Salud; 2010.

17. Organización Panamericana de la Salud. Prevención del suicidio: Un imperativo global [Internet]. Washington, DC: OPS; 2014 [citado 29 mar 2019]. Disponible en: https://tinyurl.com/tkvb6sp.

18. Campbell F, Biggs K, Aldiss SK, O'Neill PM, Clowes M, McDonagh J, While A, Gibson F. Transition of care for adolescents from pediatric services to adult health services. Cochrane Database of Systematic Reviews [Internet]. 2016 [citado 29 mar 2019]. Disponible en: https://tinyurl.com/ yx2gvtgn.

19. Souza E. Masculinidade e violência no Brasil: contribuições para reflexão no campo de saúde. Ciência \& Saúde Coletiva. 2005;10(1):59-70. doi: 10.1590/S1413-81232005000100012.

20. Bell D, Beland D, Ott M. Adolescent and young adult male health: A review. Pediatrics. 2013;132(3): 535-546.

21. Argentina, Ministerio de Salud. Indicadores Básicos: Argentina 2015. Buenos Aires: MSAL, OPS; 2015.

22. Argentina, Dirección de Estadísticas e Información de Salud. Agrupamiento de causas de mortalidad por provincia de residencia, edad y sexo: Argentina, Año 2014. Boletín No 153 [Internet]. Buenos Aires: Ministerio de Salud; 2016 [citado 29 mar 2019]. Disponible en: https://tinyurl.com/ s363jrx.

23. Argentina, Ministerio de Salud, Dirección Nacional de Salud Mental y Adicciones. Mortalidad por suicidio en Argentina 2000-2013: Informe técnico preliminar. Buenos Aires: Ministerio de Salud; 2013.

24. Tomicic A, Gálvez C, Quiroz C, Martínez C, Fontbona J, Rodríguez J, Aguayo F, Rosenbaum C, Leyton F, Lagazzi I. Suicide in lesbian, gay, bisexual and trans populations: systematic review of a decade of research (2004-2014). Revista médica de Chile. 2016;144(6):723-733.

25. Garduño MA. Determinación genérica de la mortalidad masculina. Salud Problema. 2001;6(1011):29-36.

26. Argentina, Instituto Nacional de Estadística y Censos. Tablas abreviadas de mortalidad por sexo 
y edad 2008-2010: Total del país y provincias [Internet]. Buenos Aires: Instituto Nacional de Estadística y Censos; 2013 [citado 29 mar 2019]. Disponible en: https://tinyurl.com/vedhvzq.

27. Beiras A, Tagliamento G, Perucchi J, Galvao Adriao K, Toneli MJ. Derechos sexuales y reproductivos de hombres jóvenes de una ciudad del sur de Brasil: algunos apuntalamientos para los estudios de hombres y masculinidades. I CoIoquio Internacional de Estudios sobre Varones y Masculinidades y I Congreso Nacional de la Academia Mexicana de Estudios del Género de los Hombres. Violencia: ¿el juego del hombre? México; 2006.

28. Hernández Xochipa V, Palacios de Aquino N, Rodas Castillejos I. Comunicación y salud: Evaluación de procesos de interacción en los jóvenes poblanos sobre sexualidad y VIH/SIDA. I Coloquio Internacional de Estudios Sobre Varones y Masculinidades y I Congreso Nacional de la Academia Mexicana de Estudios del Género de los Hombres. Violencia: ¿el juego del hombre? México; 2006.

29. Marsiglio W. Making males mindful of their sexual and procreative identities: Using self-narratives in field settings. Perspectives on Sexual and Reproductive Health. 2003;35(5):229-233. doi: 10.1363/psrh.35.229.03.

30. Berner E, Calandra N, Corral AM, Medina V, Tarzibachi E, Vázquez S, Zingman F. Adolescencia: Un servicio amigable para la atención integral de la salud. Buenos Aires: FUSA, Hospital General de Agudos Dr. Cosme Argerich; 2009.

31. Organización Panamericana de la Salud, FUSA. Atención integral de la salud en la adolescencia: Reducción de riesgos y daños en salud reproductiva con igualdad de género. Buenos Aires: FUSA 2000; 2010.

32. Russell S, Clarke T, Clary J. Are teens "post gay"? Contemporary adolescent's sexual identity labels. Journal of Youth Adolescence. 2009;38(7):884-890.

33. Campero R. Cuerpos, poder y erotismo: Escritos inconvenientes. Montevideo: Fin de Siglo; 2013.

34. Savin-Williams RC, Diamond LM. Sexual identity trajectories among sexual-minority youth: Gender comparisons. Archives of Sexual Behavior. 2000;29(6):607-627.

35. Diamond LM. Sexual identity, attractions, and behaviors among young sexual-minority women over a two year period. Developmental Psychology. 2000;36(2):241-250.
36. Capicúa. Aportes para pensar la salud de personas trans: Actualizando el paradigma de derechos humanos en salud [Internet]. 2014. Buenos Aires: Subsecretaría de Vinculación Ciudadana, Facultad de Derecho, Universidad de Buenos Aires, Producción Horizontal; 2014 [citado 29 mar 2019]. Disponible en: https://tinyurl.com/ v5y827x.

37. Argentina, Ministerio de Salud. Atención de la salud integral de personas trans: Guía para equipos de salud [Internet]. Buenos Aires: Programa Nacional de Salud Sexual y Procreación Responsable; 2015 [citado 29 mar 2019]. Disponible en: https://tinyurl.com/zhxewpt.

38. De Keijzer B. Enfoque de género en el desarrollo de programas de salud reproductiva de adolescentes. Salud Pública de México. 2007;49:42-44.

39. Organización Mundial de la Salud. Salud para los adolescentes del mundo: Una segunda oportunidad en la segunda década [Internet]. Ginebra: Organización Mundial de la Salud; 2014 [citado 29 mar 2019]. Disponible en: https://tinyurl.com/ v8p8fs3.

40. World Health Organization, UNAIDS. Global standards for quality health-care services for adolescents: a guide to implement a standards-driven approach to improve the quality of health-care services for adolescents. Vol. 1. Geneva: OMS; 2015.

41. Fernández AM. Hacia los estudios transdisciplinarios de la subjetividad (Reformulaciones académico-políticas de la diferencia). Revista Investigaciones en Psicología. 2011;16(1):61-82.

42. Burin M, Meler I. Varones: género y subjetividad masculina. Buenos Aires: Paidós; 2000.

43. Meler I, Tajer D, (comp.). Psicoanálisis y género: debates en el foro. Buenos Aires: Lugar Editorial; 2010.

44. Bleichmar S. La subjetividad en riesgo. Buenos Aires: Topía; 2005.

45. Bleichmar S. Paradojas de la sexualidad masculina. Buenos Aires: Paidós; 2006.

46. Laws S, Mann G. ¿Así que quiere hacer participar a los niños y niñas en la investigación?: Paquete de herramientas para apoyar la participación significativa y ética de los niños y niñas en la investigación relativa a la violencia contra los niños y niñas. Estocolmo: Save the Children Suecia; 2004.

47. Campos-Ramos PC, Barbato S. Participação de crianças em pesquisas: Uma proposta conside- 
rando os avanços teórico-metodológicos. Estudos de Psicologia (Natal). 2014;19(3):189-199.

48. Vasilachis de Gialdino I, (coord.). Estrategias de investigación cualitativa. Barcelona: Gedisa; 2006.

49. Christensen $P$, James A, (eds.). Research with children: Perspectives and practices. London: Falmer Press; 2000.

50. Borzi S, Peralta L, Yacuzzi L, Cabra M. Consentimiento informado en investigaciones psicológicas con niños: antecedentes y normas éticas. VI Congreso Internacional de Investigación y Práctica Profesional en Psicología, XXI Jornadas de Investigación, X Encuentro de Investigadores en Psicología del MERCOSUR. Buenos Aires; 2014.

51. Morrow V. Ethical dilemmas in research with children and young people about their social environments. Children's Geographies. 2008;6(1):4961.

52. Tajer D, Reid G, De la Sovera S, Amore A, Barrera MI, Blanco F, Camou ML, Fronza LI, Gasteminza $F$, Jerez $N$, Kreis $M$, Manconi $M$, Ortiz
A, Petit A, Ruibal S, Speranza A, Lo Russo AJ, Lavarello ML, Arlandi N, Gutman J. Malestares entre los géneros en un colegio secundario: Nuevas libertades y nuevas conflictivas. XIV Jornadas Nacionales de Historia de las Mujeres, IX Congreso Iberoamericano de Estudios de Género. Mar del Plata: Universidad Nacional de Mar del Plata; 2019.

53. Tajer D. Construyendo una agenda de género en las políticas públicas en salud. En: Tajer D, (comp.). Género y salud: Las Políticas en acción. Buenos Aires: Lugar Editorial; 2012. p. 17-36.

54. Tajer D. El fútbol como organizador de la masculinidad. Revista La Ventana. 1998;8:248-268.

55. Pozzio M. Médic@s: otra relación posible entre perspectiva de género y políticas de salud: Perspectiva de género y políticas de salud. V Jornadas de Sociología de la Universidad Nacional de La Plata. La Plata: Facultad de Humanidades y Ciencias de la Educación; 2008.

56. D ’Elio F, Sotelo J, Santamaría C, Recchi J. Guía básica sobre diversidad sexual. Buenos Aires: Dirección de Sida y ETS, Ministerio de Salud; 2016.

\section{FORMA DE CITAR}

Tajer DJ, Reid GB, Cuadra ME, Solís M, Fernández Romeral J, Saavedra LD, Lavarello ML, Fabbio RP. Varones adolescentes en Ciudad de Buenos Aires: barreras de género en la prevención y atención de la salud. Salud Colectiva. 2019;15:e2256. doi: 10.18294/sc.2019.2256.

Recibido: 30-03-2019 | Versión final: 4-11-2019 | Aprobado: 6-12-2019

Esta obra está bajo una licencia de Creative Commons Reconocimiento-NoComercial 4.0 Internacional. Reconocimiento - Permite copiar, distribuir y comunicar públicamente la obra. A cambio, se debe reconocer y citar al autor original. No Comercial - Esta obra no puede ser utilizada con finalidades comerciales, a menos que se obtenga el permiso. 